\title{
A note on $(p, q)$-analogue type of Fubini numbers and polynomials
}

\author{
Waseem A. Khan \\ Department of Mathematics, Faculty of Science, Integral University, \\ Lucknow-226026, India \\ E-mail: waseem08_khan@rediffmail.com
}

\begin{abstract}
In this paper, we introduce a new class of $(p, q)$-analogue type of Fubini numbers and polynomials and investigate some properties of these polynomials. We establish summation formulas of these polynomials by summation techniques series. Furthermore, we consider some relationships for $(p, q)$-Fubini polynomials associated with $(p, q)$-Bernoulli polynomials, $(p, q)$-Euler polynomials and $(p, q)$-Genocchi polynomials and $(p, q)$-Stirling numbers of the second kind.
\end{abstract}

Keywords: $(p, q)$-calculus, $(p, q)$-Bernoulli polynomials, $(p, q)$-Euler polynomials, $(p, q)$-Genocchi polynomials, $(p, q)$-Fubini numbers and polynomials, $(p, q)$ Stirling numbers of the second kind.

2010 Mathematics Subject Classification.: 11B 68, 11B73, 11B75, 11B83, 05A30.

\section{Introduction}

During the last two decades, the theory of $(p, q)$-calculus has been discussed and investigated extensively by many mathematicians and also physicists. For example Corcino [3] worked on the $(p, q)$-generalization of the binomial coefficients and also provided several useful properties parallel to those of the ordinary and $q$-binomial coefficients. Duran et al. [5-8] considered $(p, q)$-extensions of Bernoulli polynomials, Euler polynomials and Genocchi polynomials and obtained the $(p, q)$-analogues of familiar earlier formulas and identities. Milovanovic et al. [15] introduced a new generalization of Beta functions based on $(p, q)$-numbers and committed the integral modification of the generalized Bernstien polynomials. Sadjang [20] developed several properties of the $(p, q)$-derivatives and the $(p, q)$-integrals and as an application gave two $(p, q)$-Taylor formulas for polynomials.

Throughout this presentation, we use the following standard notions $\mathbb{N}=\{1,2, \cdots\}$, $\mathbb{N}_{0}=\{0,1,2, \cdots\}=\mathrm{N} \cup\{0\}, \mathbb{Z}^{-}=\{-1,-2, \cdots\}$. Also as usual $\mathbb{Z}$ denotes the set of integers, $\mathbb{R}$ denotes the set of real numbers and $\mathbb{C}$ denotes the set of complex numbers.

The $(p, q)$-numbers are defined as

$$
[n]_{p, q}=p^{n-1}+p^{n-2} q+p^{n-3} q^{2}+\cdots+p q^{n-2}+q^{n-1}=\frac{p^{n}-q^{n}}{p-q}
$$

We can write easily that $[n]_{p, q}=p^{n-1}[n]_{q / p}$, where $[n]_{q / p}$ is the $q$-number in $q$-calculus given by $[n]_{q / p}=\frac{(q / p)^{n}-1}{(q / p)-1}$. Thereby this implies that $(p, q)$-numbers and q-numbers are different, that is, we cannot obtain $(p, q)$-numbers just by substituting $q$ by $q / p$ in the definition of $q$-numbers. In the case of $p=1,(p, q)$-numbers reduce to $q$ numbers, (see $[9,10])$.

The $(p, q)$-derivative of a function $f$ with respect to $x$ is defined by

$$
D_{p, q} f(x)=D_{p, q} f(x)=\frac{f(p x)-f(q x)}{(p-q) x},(x \neq 0)
$$


and $\left(D_{p, q} f(0)\right)=f^{\prime}(0)$, provided that $\mathrm{f}$ is differentiable at 0 . The number $(p, q)$ derivative operator holds the following properties

$$
D_{p, q}(f(x) g(x))=g(p(x)) D_{p, q} f(x)+f(q x) D_{p, q} g(x),
$$

and

$$
D_{p, q}\left(\frac{f(x)}{g(x)}\right)=\frac{g(q x) D_{p, q} f(x)-f(q x) D_{p, q} g(x)}{g(p x) g(q x)} .
$$

The $(p, q)$-analogue of $(x+a)^{n}$ is given by

$$
\begin{gathered}
(x+a)_{p, q}^{n}=(x+a)(p x+a q) \cdots\left(p^{n-2} x+a q^{n-2}\right)\left(p^{n-1} x+a q^{n-1}\right), n \geq 1 \\
=\sum_{k=0}^{n}\left(\begin{array}{l}
n \\
k
\end{array}\right)_{p, q} p^{\left(\begin{array}{c}
n \\
2
\end{array}\right)} q^{\left(\begin{array}{c}
n-k \\
2
\end{array}\right)} x^{k} a^{n-k},
\end{gathered}
$$

where the $(p, q)$-Gauss Binomial coefficients $\left(\begin{array}{l}n \\ k\end{array}\right)_{p, q}$ and $(p, q)$-factorial $[n]_{p, q}$ ! are defined by

$$
\left(\begin{array}{l}
n \\
k
\end{array}\right)_{p, q}=\frac{[n]_{p, q} !}{[n-k]_{p, q ![k] p, q} !}(n \geq k) \text { and }[n]_{p, q} !=[n]_{p, q} \cdots[2]_{p, q}[1]_{p, q},(n \in \mathbb{N}) .
$$

The $(p, q)$-exponential function are defined by

$$
e_{p, q}(x)=\sum_{n=0}^{\infty} \frac{p^{\left(\begin{array}{c}
n \\
2
\end{array}\right)} x^{n}}{[n]_{p, q} !} \text { and } E_{p, q}(x)=\sum_{n=0}^{\infty} \frac{q^{\left(\begin{array}{c}
n \\
2
\end{array}\right)} x^{n}}{[n]_{p, q} !}
$$

holds the identities

$$
e_{p, q}(x) E_{p, q}(-x)=1 \text { and } e_{p^{-} q^{-}}(x)=E_{p, q}(x),
$$

and have the $(p, q)$-derivatives

$$
D_{p, q} e_{p, q}(x)=e_{p, q}(p x) \text { and } D_{p, q} E_{p, q}(x)=E_{p, q}(q x) .
$$

The definition $(p, q)$-integral is defined by

$$
\int_{0}^{a} f(x) d_{p, q} x=(p-q) a \sum_{k=0}^{\infty} \frac{p^{k}}{q^{k+1}} f\left(a \frac{p^{k}}{q^{k+1}}\right),
$$

in conjunction with

$$
\int_{a}^{b} f(x) d_{p, q} x=\int_{0}^{b} f(x) d_{p, q} x-\int_{0}^{a} f(x) d_{p, q} x, \quad \text { (see [20]). }
$$

The generalized $(p, q)$-Bernoulli polynomials, the generalized $(p, q)$-Euler polynomials and the generalized $(p, q)$-Genocchi polynomials are defined by means of the following generating function as follows (see [3-10, 13-15, 18, 20]):

$$
\begin{aligned}
& \left(\frac{t}{e_{p, q}(t)-1}\right)^{\alpha} e_{p, q}(x t) E_{p, q}(y t)=\sum_{n=0}^{\infty} B_{n}^{(\alpha)}(x, y: p, q) \frac{t^{n}}{[n]_{p, q} !},|t|<2 \pi \\
& \left(\frac{2}{e_{p, q}(t)+1}\right)^{\alpha} e_{p, q}(x t) E_{p, q}(y t)=\sum_{n=0}^{\infty} E_{n}^{(\alpha)}(x, y: p, q) \frac{t^{n}}{[n]_{p, q} !},|t|<\pi
\end{aligned}
$$

and

$$
\left(\frac{2 t}{e_{p, q}(t)+1}\right)^{\alpha} e_{p, q}(x t) E_{p, q}(y t)=\sum_{n=0}^{\infty} G_{n}^{(\alpha)}(x, y: p, q) \frac{t^{n}}{[n]_{p, q} !},|t|<\pi .
$$

It is clear that

$$
B_{n}^{(\alpha)}(0,0: p, q)=B_{n}^{(\alpha)}(p, q), E_{n}^{(\alpha)}(0,0: p, q)=E_{n}^{(\alpha)}(p, q),
$$


and

$$
G_{n}^{(\alpha)}(0,0: p, q)=G_{n}^{(\alpha)}(p, q)(n \in \mathbb{N}) .
$$

Geometric polynomials (also known as Fubini polynomials) are defined as follows (see [1]):

$$
F_{n}(x)=\sum_{k=0}^{n}\left\{\begin{array}{l}
n \\
k
\end{array}\right\} k ! x^{k}
$$

where $\left\{\begin{array}{l}n \\ k\end{array}\right\}$ is the Stirling number of the second kind (see [11]).

For $x=1$ in (1.12), we get $n^{\text {th }}$ Fubini number (ordered Bell number or geometric number) $F_{n}[2,11,12,21]$ is defined by

$$
F_{n}(1)=F_{n}=\sum_{k=0}^{n}\left\{\begin{array}{l}
n \\
k
\end{array}\right\} k !
$$

The exponential generating functions of geometric polynomials is given by (see [1]):

$$
\frac{1}{1-x\left(e^{t}-1\right)}=\sum_{n=0}^{\infty} F_{n}(x) \frac{t^{n}}{n !},
$$

and related to the geometric series (see [1]):

$$
\left(x \frac{d}{d x}\right)^{m} \frac{1}{1-x}=\sum_{k=0}^{\infty} k^{m} x^{k}=\frac{1}{1-x} F_{m}\left(\frac{x}{1-x}\right),|x|<1 .
$$

Let us give a short list of these polynomials and numbers as follows:

$F_{0}(x)=1, F_{1}(x)=x, F_{2}(x)=x+2 x^{2}, F_{3}(x)=x+6 x^{2}+6 x^{3}, F_{4}(x)=x+14 x^{2}+36 x^{3}+24 x^{4}$, and

$$
F_{0}=1, F_{1}=1, F_{2}=3, F_{3}=13, F_{4}=75 .
$$

Geometric and exponential polynomials are connected by the relation (see [1]):

$$
F_{n}(x)=\int_{0}^{\infty} \phi_{n}(x) e^{-\lambda} d \lambda
$$

The goal of this paper as follows: In section 2, we consider generating functions for $(p, q)$-analogue type of Fubini numbers and polynomials and give some properties of these numbers and polynomials. In section 3, we derive summation formulas for $(p, q)$-analogue type of Fubini numbers and polynomials. In Section 4 , we construct a relationships for $(p, q)$-Fubini polynomials associated with $(p, q)$-Bernoulli polynomials, $(p, q)$-Euler polynomials and $(p, q)$-Genocchi polynomials and $(p, q)$-Stirling numbers of the second kind.

\section{2. $(p, q)$-analogue type of Fubini numbers and polynomials}

In this section, we introduce $(p, q)$-Fubini polynomials and obtain some basic properties which gives us new formula for $F_{n}(x, y ; z: p, q)$.

Definition 2.1. Let $p, q \in \mathbb{C}$ with $0<|q|<|p| \leq 1$, the $(p, q)$-Fubini polynomials $F_{n}(x, y ; z: p, q)$ are defined by means of the following generating function:

$$
\frac{1}{1-z\left(e_{p, q}(t)-1\right)} e_{p, q}(x t) E_{p, q}(y t)=\sum_{n=0}^{\infty} F_{n}(x, y ; z: p, q) \frac{t^{n}}{[n]_{p, q} !} .
$$

It is easily seen from definition (2.1), we have

$$
F_{n}(x, y ; 1: p, q)=F_{n}(x, y: p, q), F_{n}(0,0 ; 1: p, q)=F_{n}(p, q),
$$


where $F_{n}(p, q)$ are called the $(p, q)$-Fubini numbers.

Obviously that

$$
\begin{gathered}
F_{n}(0,0 ; z: p, q)=F_{n}(z: p, q), \\
\left.F_{n}(x, y ; z: p, q)\right|_{p=1}=F_{n}(x, y ; z: q), \\
\lim _{q \longrightarrow 1_{p=1}^{-}} F_{n}(x, 0: p, q)=F_{n}(x ; z), \quad(\text { see }[1]) \\
\lim _{q \longrightarrow 1_{p=1}^{-}} F_{n}(0, o ; z: p, q)=F_{n}(z), \quad(\text { see }[1,12]) .
\end{gathered}
$$

Theorem 2.1. The following series representation for the $(p, q)$-Fubini polynomials $F_{n}(x, y ; z: p, q)$ holds true:

$$
F_{n}(x, y ; z: p, q)=\sum_{m=0}^{n}\left(\begin{array}{l}
n \\
m
\end{array}\right)_{p, q} F_{n-m}(p, q)(\lambda)(x+y)_{p, q}^{m} .
$$

Proof. Using equation (1.4) and (2.1) in the l.h.s. of equation (2.1) and then applying the Cauchy product rule and equating the coefficients of same powers of $t$ in both sides of resultant equation, we get representation (2.3).

Theorem 2.2. The following summation formula for the $(p, q)$-Fubini polynomials $F_{n}(x, y ; z: p, q)$ holds true:

$$
\begin{gathered}
F_{n}(x, y ; 0: p, q)=\sum_{m=0}^{n}\left(\begin{array}{c}
n \\
m
\end{array}\right)_{p, q} p^{\left(\begin{array}{c}
m \\
2
\end{array}\right) q^{\left(\begin{array}{c}
n-m \\
2
\end{array}\right)} x^{m} y^{n-m} .} \\
F_{n}(x, y ; z: p, q)=\sum_{m=0}^{n}\left(\begin{array}{c}
n \\
m
\end{array}\right)_{p, q} p^{\left(\begin{array}{c}
n-m \\
2
\end{array}\right)} F_{m}(0, y ; z: p, q) x^{n-m} . \\
F_{n}(x, y ; z: p, q)=\sum_{m=0}^{n}\left(\begin{array}{c}
n \\
m
\end{array}\right)_{p, q} q^{\left(\begin{array}{c}
n-m \\
2
\end{array}\right)} F_{m}(x, 0 ; z: p, q) y^{n-m} .
\end{gathered}
$$

Proof. Suitably using equations (1.4)-(1.6) in generating function (2.1) to get three different form. Further making use of the Cauchy product rule in the resultant expressions and then comparing the like powers of $t$ in the both sides of resultant equation, we find formulas (2.4)-(2.6).

Theorem 2.3. For $n \geq 0$, the following formula for $(p, q)$-type Fubini polynomials holds true:

$$
(x+y)_{p, q}^{n}=F_{n}(x, y ; z: p, q)-z F_{n}(x+1, y ; z: p, q)+z F_{n}(x, y ; z: p, q) .
$$

Proof. We begin with the definition (2.1) and write

$$
\begin{aligned}
& e_{p, q}(x t) E_{p, q}(y t)=\frac{1-z\left(e_{p, q}(t)-1\right)}{1-z\left(e_{p, q}(t)-1\right)} e_{p, q}(x t) E_{p, q}(y t) \\
= & \frac{e_{p, q}(x t) E_{p, q}(y t)}{1-z\left(e^{t}-1\right)}-\frac{z\left(e_{p, q}(t)-1\right)}{1-z\left(e_{p, q}(t)-1\right)} e_{p, q}(x t) E_{p, q}(y t) .
\end{aligned}
$$

Then using the definition of (1.4) and (2.1), we have

$\sum_{n=0}^{\infty}(x+y)_{p, q}^{n} \frac{t^{n}}{[n]_{p, q} !}=\sum_{n=0}^{\infty}\left[F_{n}(x, y ; z: p, q)-z F_{n}(x+1, y ; z: p, q)+z F_{n}(x, y ; z: p, q)\right] \frac{t^{n}}{[n]_{p, q} !}$.

Finally, comparing the coefficients of $\frac{t^{n}}{n !}$, we get (2.7). 
Theorem 2.4. The following formula for $(p, q)$-type Fubini polynomials holds true:

$$
z F_{n}(x+1, y ; z: p, q)=(1+z) F_{n}(x, y ; z: p, q)-(x+y)_{p, q}^{n}
$$

Proof. From (2.1), we have

$$
\begin{aligned}
\sum_{n=0}^{\infty}\left[F_{n}(x+1, y ; z\right. & \left.: p, q)-F_{n}(x, y ; z: p, q)\right] \frac{t^{n}}{[n]_{p, q} !}=\frac{e_{p, q}(x t) E_{p, q}(y t)}{1-z\left(e_{p, q}(t)-1\right)}\left(e_{p, q}(t)-1\right) \\
& =\frac{1}{z}\left[\frac{e_{p, q}(x t) E_{p, q}(y t)}{1-z\left(e_{p, q}(t)-1\right)}-e_{p, q}(x t) E_{p, q}(y t)\right] \\
& =\frac{1}{z} \sum_{n=0}^{\infty}\left[F_{n}(x, y ; z: p, q)-(x+y)_{p, q}^{n}\right] \frac{t^{n}}{[n]_{p, q} !} .
\end{aligned}
$$

Comparing the coefficients of $\frac{t^{n}}{n !}$ on both sides, we obtain (2.8).

Theorem 2.5. The following recursive formulas for the $(p, q)$-Fubini polynomials $F_{n}(x, y ; z: p, q)$ holds true:

$$
\begin{aligned}
& D_{p, q ; x} F_{n}(x, y ; z: p, q)=[n]_{p, q} F_{n-1}(p x, y ; z: p, q), \\
& D_{p, q ; y} F_{n}(x, y ; z: p, q)=[n]_{p, q} F_{n-1}(x, q y ; z: p, q) .
\end{aligned}
$$

Proof. Differentiating generating function (2.1) with respect to $x$ and $y$ with the help of equation (1.7) and then simplifying with the help of the Cauchy product rule formulas (2.9) and (2.10) are obtained.

Theorem 2.6. The following $(p, q)$-integral is valid

$$
\begin{aligned}
\int_{a}^{b} F_{n}(x, y ; z: p, q) d_{p, q} x & =p \frac{F_{n+1}\left(\frac{b}{p}, y ; z: p, q\right)-F_{n+1}\left(\frac{a}{p}, y ; z: p, q\right)}{[n+1]_{p, q}}, \\
\int_{a}^{b} F_{n}(x, y ; z: p, q) d_{p, q} y & =p \frac{F_{n+1}\left(x, \frac{b}{q} ; z: p, q\right)-F_{n+1}\left(x, \frac{a}{q} ; z: p, q\right)}{[n+1]_{p, q}} .
\end{aligned}
$$

Proof. Since

$$
\int_{a}^{b} \frac{\delta}{\delta_{p, q} x} F_{n}(x, y ; z: p, q) d_{p, q} x=f(b)-f(a)(\text { see }[20]),
$$

in terms of equation (2.9) and equations (1.7) and (1.8), we arrive at the asserted result

$$
\begin{gathered}
\int_{a}^{b} \frac{\delta}{\delta_{p, q} x} F_{n}(x, y ; z: p, q) d_{p, q} x=\frac{p}{[n+1]_{p, q}} \int_{a}^{b} F_{n}\left(\frac{x}{p}, y ; z: p, q\right) d_{p, q} x \\
=p \frac{F_{n+1}\left(\frac{b}{p}, y ; z: p, q\right)-F_{n+1}\left(\frac{b}{p}, y ; z: p, q\right)}{[n+1]_{p, q}} .
\end{gathered}
$$

The other can be shown using similar method. Therefore, the complete the proof of this theorem.

\section{Main results}

First, we prove the following result involving the $(p, q)$-Fubini polynomials $F_{n}(x, y ; z$ : $p, q)$ by using series rearrangement techniques and considered its special case: 
Theorem 3.1. The following summation formula for $(p, q)$-type Fubini polynomials $F_{n}(x, y ; z: p, q)$ holds true:

$F_{k+l}(w, y ; z: p, q)=\sum_{n, s=0}^{k, l}\left(\begin{array}{l}k \\ n\end{array}\right)_{p, q}\left(\begin{array}{l}l \\ s\end{array}\right)_{p, q} p^{\left(\begin{array}{c}n+s \\ 2\end{array}\right)}(w-x)^{n+s} F_{k+l-n-s}(x, y ; z: p, q)$.

Proof. Replacing $t$ by $t+u$ in (2.1) and then using the formula [19,p.52(2)]:

$$
\sum_{N=0}^{\infty} f(N) \frac{(x+y)^{N}}{N !}=\sum_{n, m=0}^{\infty} f(n+m) \frac{x^{n}}{n !} \frac{y^{m}}{m !}
$$

in the resultant equation, we find the following generating function for the $(p, q)$-type Fubini polynomials $F_{n}(x, y ; z: p, q)$ :

$$
\begin{gathered}
\frac{1}{1-z\left(e_{p, q}(t+u)-1\right)} E_{p, q}(y(t+u)) \\
\left.=e_{p, q}(-x(t+u)) \sum_{k, l=0}^{\infty} F_{k+l}(x, y ; z: p, q) \frac{t^{k}}{[k]_{p, q} !} \frac{u^{l}}{[l]_{p, q} !}, \text { (see }[16,17]\right) .
\end{gathered}
$$

Replacing $x$ by $w$ in the above equation and equating the resultant equation to the above equation, we find

$$
\begin{gathered}
e_{p, q}((w-x)(t+u)) \sum_{k, l=0}^{\infty} F_{k+l}(x, y ; z: p, q) \frac{t^{k}}{[k]_{p, q} !} \frac{u^{l}}{[l]_{p, q} !} \\
=\sum_{k, l=0}^{\infty} F_{k+l}(w, y ; z: p, q) \frac{t^{k}}{[k]_{p, q} !} \frac{u^{l}}{[l]_{p, q} !}
\end{gathered}
$$

On expanding exponential function (3.4) gives

$$
\begin{gathered}
\sum_{N=0}^{\infty} \frac{[(w-x)(t+u)]^{N} p^{\left(\begin{array}{c}
N \\
2
\end{array}\right)}}{[N]_{p, q} !} \sum_{k, l=0}^{\infty} F_{k+l}(x, y ; z: p, q) \frac{t^{k}}{[k]_{p, q} !} \frac{u^{l}}{[l]_{p, q} !} \\
=\sum_{k, l=0}^{\infty} F_{k+l}(w, y ; z: p, q) \frac{t^{k}}{[k]_{p, q} !} \frac{u^{l}}{[l]_{p, q} !},
\end{gathered}
$$

which on using formula (3.2) in the first summation on the left hand side becomes

$$
\begin{gathered}
\sum_{n, s=0}^{\infty} \frac{(w-x)^{n+s} t^{n} u^{s} p^{\left(\begin{array}{c}
n+s \\
2
\end{array}\right)}}{[n]_{p, q} ![s]_{p, q} !} \sum_{k, l=0}^{\infty} F_{k+l}(x, y ; z: p, q) \frac{t^{k}}{[k]_{p, q} !} \frac{u^{l}}{[l]_{p, q} !} \\
=\sum_{k, l=0}^{\infty} F_{k+l}(w, y ; z: p, q) \frac{t^{k}}{[k]_{p, q} !} \frac{u^{l}}{[l]_{p, q} !} .
\end{gathered}
$$

Now replacing $k$ by $k-n, l$ by $l-s$ and using the lemma ([19, p.100(1)]):

$$
\sum_{k=0}^{\infty} \sum_{n=0}^{\infty} A(n, k)=\sum_{k=0}^{\infty} \sum_{n=0}^{k} A(n, k-n)
$$

in the l.h.s. of (3.6), we find

$$
\sum_{k, l=0}^{\infty} \sum_{n, s=0}^{k, l} \frac{(w-x)^{n+s} p^{\left(\begin{array}{c}
n+s \\
2
\end{array}\right)}}{[n]_{p, q} ![s]_{p, q} !} F_{k+l-n-s}\left(x, y ; z^{\prime \prime} p, q\right) \frac{t^{k}}{(k-n)_{p, q} !} \frac{u^{l}}{(l-s)_{p, q} !}
$$




$$
=\sum_{k, l=0}^{\infty} F_{k+l}(w, y ; z: p, q) \frac{t^{k}}{[k]_{p, q} !} \frac{u^{l}}{[l]_{p, q} !} .
$$

Finally, on equating the coefficients of the like powers of $t$ and $u$ in the above equation, we get the assertion (3.1) of Theorem 3.1.

Remark 3.1. Taking $l=0$ in assertion (3.1) of Theorem 3.1, we deduce the following consequence of Theorem 3.1 .

Corollary 3.1. The following summation formula for $(p, q)$-type Fubini polynomials $F_{n}(x, y ; z: p, q)$ holds true:

$$
F_{k}(w, y ; z: p, q)=\sum_{n=0}^{k}\left(\begin{array}{c}
k \\
n
\end{array}\right)_{p, q} p^{\left(\begin{array}{c}
n+s \\
2
\end{array}\right)}(w-x)^{n} F_{k-n}(x, y ; z: p, q) .
$$

Remark 3.2. Replacing $w$ by $w+x$ in (3.9), we obtain

$$
F_{k}(w+x, y ; z: p, q)=\sum_{n=0}^{k}\left(\begin{array}{l}
k \\
n
\end{array}\right)_{p, q} p^{\left(\begin{array}{c}
n+s \\
2
\end{array}\right)} w^{n} F_{k-n}(x, y ; z: p, q) .
$$

Theorem 3.2. The following summation formula for $(p, q)$-type Fubini polynomials $F_{n}(x, y ; z: p, q)$ holds true:

$$
\begin{gathered}
F_{n}(w, u ; z: p, q) F_{m}(W, U ; Z: p, q)=\sum_{r, k=0}^{n, m}\left(\begin{array}{c}
n \\
r
\end{array}\right)_{p, q}\left(\begin{array}{c}
m \\
k
\end{array}\right)_{p, q}(w-x+u-y)_{p, q}^{r} \\
\times F_{n-r}(x, y ; z: p, q)(W-X+U-Y)_{p, q}^{k} F_{m-k}(X, Y ; Z: p, q) .
\end{gathered}
$$

Proof. Consider the product of the $(p, q)$-type Fubini polynomials, we can be written as generating function (2.1) in the following form:

$$
\begin{gathered}
\frac{1}{1-z\left(e_{p, q}(t)-1\right)} e_{p, q}(x t) E_{p, q}(y t) \frac{1}{1-Z\left(e_{p, q}(T)-1\right)} e_{p, q}(X T) E_{p, q}(Y T) \\
=\sum_{n=0}^{\infty} F_{n}(x, y ; z: p, q) \frac{t^{n}}{[n]_{p, q} !} \sum_{m=0}^{\infty} F_{m}(X, Y ; Z: p, q) \frac{T^{m}}{[m]_{p, q} !} .
\end{gathered}
$$

Replacing $x$ by $w, y$ by $u, X$ by $W$ and $Y$ by $U$ in (3.12) and equating the resultant to itself,

$$
\begin{aligned}
& \sum_{n=0}^{\infty} \sum_{m=0}^{\infty} F_{n}(w, u ; z: p, q) F_{m}(W, U ; Z: p, q) \frac{t^{n}}{[n]_{p, q} !} \frac{T^{m}}{[m]_{p, q} !} \\
= & e_{p, q}((w-x) t) E_{p, q}((u-y) t) e_{p, q}((W-X) T) E_{p, q}((U-Y) t) \\
& \times \sum_{n=0}^{\infty} \sum_{m=0}^{\infty} F_{n}(x, y ; z: p, q) F_{m}(X, Y ; Z: p, q) \frac{t^{n}}{[n]_{p, q} !} \frac{T^{m}}{[m]_{p, q} !},
\end{aligned}
$$

which on using the generating function (3.7) in the exponential on the r.h.s., becomes

$$
\begin{aligned}
& \sum_{n=0}^{\infty} \sum_{m=0}^{\infty} F_{n}(w, u ; z: p, q) F_{m}(W, U ; Z: p, q) \frac{t^{n}}{[n]_{p, q} !} \frac{T^{m}}{[m]_{p, q} !} \\
& \quad=\sum_{n, r=0}^{\infty}(w-x+u-y)_{p, q}^{r} F_{n}(x, y ; z) \frac{t^{n+r}}{[n]_{p, q} ![r]_{p, q} !} \\
& \times \sum_{m, k=0}^{\infty}(W-X+U-Y)_{p, q H}^{k} F_{m}(X, Y ; Z) \frac{T^{m+k}}{[m]_{p, q} ![k]_{p, q} !} .
\end{aligned}
$$


Finally, replacing $n$ by $n-r$ and $m$ by $m-k$ and using equation (3.7) in the r.h.s. of the above equation and then equating the coefficients of like powers of $t$ and $T$, we get assertion (3.11) of Theorem 3.2.

Remark 3.3. Replacing $u$ by $y$ and $U$ by $Y$ in assertion (3.11) of Theorem 3.2, we deduce the the following consequence of Theorem 3.2.

Corollary 3.2. The following summation formula for $(p, q)$-type Fubini polynomials $F_{n}(x, y ; z: p, q)$ holds true:

$$
\begin{gathered}
F_{n}(w, y ; z: p, q) F_{m}(W, Y ; Z: p, q)=\sum_{r, k=0}^{n, m}\left(\begin{array}{c}
n \\
r
\end{array}\right)_{p, q}\left(\begin{array}{c}
m \\
k
\end{array}\right)_{p, q}(w-x)_{p, q}^{r} F_{n-r}(x, u ; z: p, q) \\
\times(W-X)_{p, q}^{k} F_{m-k}(X, U ; Z: p, q) .
\end{gathered}
$$

Theorem 3.3. The following summation formula for $(p, q)$-type Fubini polynomials $F_{n}(x, y ; z: p, q)$ holds true:

$$
F_{n}(x+1, y ; z: p, q)=\sum_{r=0}^{n}\left(\begin{array}{l}
n \\
r
\end{array}\right)_{p, q} F_{n-r}(x, y ; z: p, q) .
$$

Proof. Using the generating function (2.1), we have

$$
\begin{gathered}
\sum_{n=0}^{\infty} F_{n}(x+1, y ; z: p, q) \frac{t^{n}}{[n]_{p, q} !}-\sum_{n=0}^{\infty} F_{n}(x, y ; z: p, q) \frac{t^{n}}{[n]_{p, q} !} \\
=\left(\frac{1}{1-z\left(e_{p, q}(t)-1\right)}\right)\left(e_{p, q}(t)-1\right) e_{p, q}(x t) E_{p, q}(y t) \\
=\sum_{n=0}^{\infty} F_{n}(x, y ; z: p, q) \frac{t^{n}}{[n]_{p, q} !}\left(\sum_{r=0}^{\infty} \frac{t^{r}}{[r]_{p, q} !}-1\right) \\
=\sum_{n=0}^{\infty} F_{n}(x, y ; z: p, q) \frac{t^{n}}{[n]_{p, q} !} \sum_{r=0}^{\infty} \frac{t^{r}}{[r]_{p, q} !}-\sum_{n=0}^{\infty} F_{n}(x, y ; z: p, q) \frac{t^{n}}{[n]_{p, q} !} \\
=\sum_{n=0}^{\infty} \sum_{r=0}^{n}\left(\begin{array}{c}
n \\
r
\end{array}\right)_{p, q} F_{n-r}(x, y ; z: p, q) \frac{t^{n}}{[n]_{p, q} !}-\sum_{n=0}^{\infty} F_{n}(x, y ; z: p, q) \frac{t^{n}}{[n]_{p, q} !}
\end{gathered}
$$

Finally, equating the coefficients of the like powers of $t$ on both sides, we get $(3.15)$.

Theorem 3.4. For $n \geq 0$ and $z_{1} \neq z_{2}$, the following formula for $(p, q)$-type HermiteFubini polynomials holds true:

$$
\begin{gathered}
\left(\begin{array}{c}
n \\
k
\end{array}\right)_{p, q} F_{n-k}\left(x_{1}, y_{1} ; z_{1}: p, q\right) F_{k}\left(x_{2}, y_{2} ; z_{2}: p, q\right) \\
=\frac{z_{2} F_{n}\left(x_{1}+x_{2}, y_{1}+y_{2} ; z_{2}: p, q\right)-z_{1} F_{n}\left(x_{1}+x_{2}, y_{1}+y_{2} ; z_{1}: p, q\right)}{z_{2}-z_{1}} .
\end{gathered}
$$

Proof. The products of $(2.1)$ can be written as

$$
\begin{gathered}
\sum_{n=0}^{\infty} \sum_{k=0}^{\infty} F_{n}\left(x_{1}, y_{1} ; z_{1}: p, q\right) F_{k}\left(x_{2}, y_{2} ; z_{2}: p, q\right) \frac{t^{n}}{[n]_{p, q} !} \frac{t^{k}}{[k]_{p, q} !} \\
=\frac{e_{p, q}\left(x_{1} t\right) E_{p, q}\left(y_{1} t\right)}{1-z_{1}\left(e_{p, q}(t)-1\right)} \frac{e_{p, q}\left(x_{2} t\right) E_{p, q}\left(y_{2} t\right)}{1-z_{2}\left(e_{p, q}(t)-1\right)}
\end{gathered}
$$




$$
\begin{aligned}
& \sum_{n=0}^{\infty}\left(\sum_{k=0}^{n}\left(\begin{array}{c}
n \\
k
\end{array}\right)_{p, q} F_{n-k}\left(x_{1}, y_{1} ; z_{1}: p, q\right) F_{k}\left(x_{2}, y_{2} ; z_{2}: p, q\right)\right) \frac{t^{n}}{[n]_{p, q} !} \\
= & \frac{z_{2}}{z_{2}-z_{1}} \frac{e_{p, q}\left[\left(x_{1}+x_{2}\right) t\right] E_{p, q}\left[\left(y_{1}+y_{2}\right) t\right]}{1-z_{1}\left(e_{p, q}(t)-1\right)}-\frac{z_{1}}{z_{2}-z_{1}} \frac{e_{p, q}\left[\left(x_{1}+x_{2}\right) t\right] E_{p, q}\left[\left(y_{1}+y_{2}\right) t\right]}{1-z_{2}\left(e_{p, q}(t)-1\right)} \\
= & \left(\frac{z_{2} F_{n}\left(x_{1}+x_{2}, y_{1}+y_{2} ; z_{2}: p, q\right)-z_{1} F_{n}\left(x_{1}+x_{2}, y_{1}+y_{2} ; z_{1}: p, q\right)}{z_{2}-z_{1}}\right) \frac{t^{n}}{[n]_{p, q} !} .
\end{aligned}
$$

By equating the coefficients of $\frac{t^{n}}{[n]_{p, q} !}$ on both sides, we get (3.16).

Theorem 3.5. The following relation for the $(p, q)$-type Fubini polynomials $F_{n}(x, y ; z$ : $p, q$ ) holds true:

$$
(1+z) F_{n}(x, y ; z: p, q)=z \sum_{k=0}^{n}\left(\begin{array}{c}
n \\
k
\end{array}\right)_{p, q} F_{n-k}(x, y ; z: p, q)+(x+y)_{p, q}^{n} .
$$

Proof. Consider the following identity

$$
\frac{1+z}{\left(1-z\left(e_{p, q}(t)-1\right)\right) z e_{p, q}(t)}=\frac{1}{1-z\left(e_{p, q}(t)-1\right)}+\frac{1}{z e_{p, q}(t)}
$$

Evaluating the following fraction using above identity, we find

$$
\begin{gathered}
\frac{(1+z) e_{p, q}(x t) E_{p, q}(y t)}{\left(1-z\left(e_{p, q}(t)-1\right)\right) z e_{p, q}(t)}=\frac{e_{p, q}(x t) E_{p, q}(y t)}{1-z\left(e_{p, q}(t)-1\right)}+\frac{e_{p, q}(x t) E_{p, q}(y t)}{z e_{p, q}(t)} \\
(1+z) \sum_{n=0}^{\infty} F_{n}(x, y ; z: p, q) \frac{t^{n}}{[n]_{p, q} !} \\
=z \sum_{n=0}^{\infty} F_{n}(x, y ; z: p, q) \frac{t^{n}}{[n]_{p, q} !} \sum_{k=0}^{\infty} \frac{t^{k}}{[k]_{p, q} !}-\sum_{n=0}^{\infty}(x+y)_{p, q}^{n} \frac{t^{n}}{[n]_{p, q} !} .
\end{gathered}
$$

Applying the Cauchy product rule in the above equation and then equating the coefficients of like powers of $t$ in both sides of the resultant equation, assertion (3.17) follows.

4. Relationship between $(p, q)$-Bernoulli, $(p, q)$-Euler and $(p, q)$-Genocchi polynomials and $(p, q)$-Stirling numbers of the second kind,

In this section, we prove some relationships for $(p, q)$ - type Fubini polynomials related to $(p, q)$-Bernoulli polynomials, $(p, q)$-Euler polynomials and $(p, q)$-Genocchi polynomials and $(p, q)$-Stirling numbers of the second kind. We start a following theorem.

Theorem 4.1. Each of the following relationships holds true:

$$
\begin{aligned}
& F_{n}(x, y ; z: p, q) \\
& =\sum_{s=0}^{n+1}\left(\begin{array}{l}
n+1 \\
s
\end{array}\right)_{p, q}\left[\sum_{k=0}^{s}\left(\begin{array}{l}
s \\
k
\end{array}\right)_{p, q} B_{s-k}(x ; p, q) p^{\left.\left(\begin{array}{l}
k \\
2
\end{array}\right)-B_{s}(x ; p, q)\right]} \frac{F_{n+1-s}(0, y ; z: p, q)}{[n+1]_{p, q}},\right.
\end{aligned}
$$

where $B_{n}(x ; p, q)$ is $(p, q)$-Bernoulli polynomials. 
Proof. By using definition (2.1), we have

$$
\begin{aligned}
& \left(\frac{1}{1-z\left(e_{p, q}(t)-1\right)}\right) e_{p, q}(x t) E_{p, q}(y t) \\
& =\left(\frac{1}{1-z\left(e_{p, q}(t)-1\right)}\right) \frac{t}{e_{p, q}(t)-1} \frac{e_{p, q}(t)-1}{t} e_{p, q}(x t) E_{p, q}(y t) \\
& =\frac{1}{t} \sum_{n=0}^{\infty}\left(\sum_{k=0}^{s}\left(\begin{array}{c}
s \\
k
\end{array}\right)_{p, q} B_{s-k}(x ; p, q) p^{\left.\left(\begin{array}{c}
k \\
2
\end{array}\right)\right)} \frac{t^{s}}{[s]_{p, q} !} \sum_{n=0}^{\infty} F_{n}(0, y ; z: p, q) \frac{t^{n}}{[n]_{p, q} !}\right. \\
& -\frac{1}{t} \sum_{s=0}^{\infty} B_{s}(x ; p, q) \frac{t^{s}}{[s]_{p, q} !} \sum_{n=0}^{\infty} F_{n}(0, y ; z: p, q) \frac{t^{n}}{[n]_{p, q} !} \\
& =\frac{1}{t} \sum_{n=0}^{\infty}\left[\sum_{s=0}^{n}\left(\begin{array}{c}
n \\
s
\end{array}\right)_{p, q} \sum_{k=0}^{s}\left(\begin{array}{c}
s \\
k
\end{array}\right) B_{p, q} B_{s-k}(x ; p, q) p\left(\begin{array}{l}
k \\
2
\end{array}\right)\right] F_{n-s}(0, y ; z: p, q) \frac{t^{n}}{[n]_{p, q} !} \\
& -\frac{1}{t} \sum_{n=0}^{\infty}\left[\sum_{s=0}^{n}\left(\begin{array}{c}
n \\
s
\end{array}\right)_{p, q} B_{s}(x ; p, q)\right]_{n-s}(0, y ; z: p, q) \frac{t^{n}}{[n]_{p, q} !} .
\end{aligned}
$$

By using Cauchy product and comparing the coefficients of $\frac{t^{n}}{[n]_{p, q} !}$, we arrive at the required result $(4.1)$.

Theorem 4.2. Each of the following relationships holds true:

$$
\begin{aligned}
& F_{n}(0, y ; z ; u: p, q) \\
& =\sum_{s=0}^{n}\left(\begin{array}{c}
n \\
s
\end{array}\right)_{p, q}\left[\sum_{k=0}^{s}\left(\begin{array}{c}
s \\
k
\end{array}\right)_{p, q} E_{s-k}(x ; p, q) p^{\left(\begin{array}{c}
k \\
2
\end{array}\right)}+E_{s}(x ; p, q)\right] \frac{F_{n-s}(0, y ; z: p, q)}{[2]_{p, q}}
\end{aligned}
$$

where $E_{n}(x ; p, q)$ is $(p, q)$-Euler polynomials.

Proof. By using definition (2.1), we have

$$
\begin{aligned}
& \left(\frac{1}{1-z\left(e_{p, q}(t)-1\right)}\right) e_{p, q}(x t) E_{p, q}(y t) \\
& =\left(\frac{1}{1-z\left(e_{p, q}(t)-1\right)}\right) \frac{[2]_{p, q}}{e_{p, q}(t)+1} \frac{e_{p, q}(t)+1}{[2]_{p, q}} e_{p, q}(x t) E_{p, q}(y t) \\
& =\frac{1}{[2]_{p, q}}\left[\sum_{n=0}^{\infty}\left(\sum_{k=0}^{n}\left(\begin{array}{c}
n \\
k
\end{array}\right)_{p, q} E_{n-k}(x ; p, q) p\left(\begin{array}{l}
k \\
2
\end{array}\right)\right) \frac{t^{n}}{[n]_{p, q} !}+\sum_{n=0}^{\infty} E_{n}(x ; p, q) \frac{t^{n}}{[n]_{p, q} !}\right] \\
& \times \sum_{n=0}^{\infty} F_{n}(0, y ; z: p, q) \frac{t^{n}}{[n]_{p, q} !} \\
& =\frac{1}{[2]_{p, q}} \sum_{n=0}^{\infty}\left[\sum_{s=0}^{n}\left(\begin{array}{c}
n \\
s
\end{array}\right)_{p, q} \sum_{k=0}^{s}\left(\begin{array}{c}
s \\
k
\end{array}\right)_{p, q} E_{s-k}(x ; p, q) p^{\left(\begin{array}{c}
k \\
2
\end{array}\right)}+\sum_{s=0}^{n}\left(\begin{array}{c}
n \\
s
\end{array}\right)_{p, q} E_{s}(x ; p, q)\right] \\
& \times F_{n-s}(0, y ; z: p, q) \frac{t^{n}}{[n]_{p, q} !} .
\end{aligned}
$$

Comparing the coefficients of $\frac{t^{n}}{[n]_{p, q} !}$, we arrive at the desired result (4.2). 
Theorem 4.3 . Each of the following relationships holds true:

$$
\begin{aligned}
& F_{n}(x, y ; z: p, q) \\
& =\sum_{s=0}^{n}\left(\begin{array}{l}
n+1 \\
s
\end{array}\right)_{p, q}\left[\sum_{k=0}^{s}\left(\begin{array}{l}
s \\
k
\end{array}\right)_{p, q} G_{s-k}(x ; p, q) p^{\left(\begin{array}{l}
k \\
2
\end{array}\right)}+G_{s}(x ; p, q)\right] \frac{F_{n-s}(0, y ; z: p, q)}{[2]_{p, q}[n+1]_{p, q}},
\end{aligned}
$$

where $G_{n}(x ; p, q)$ is $(p, q)$-Genocchi polynomials.

Proof. By using definition (2.1), we have

$$
\begin{aligned}
& \left(\frac{1}{1-z\left(e_{p, q}(t)-1\right)}\right) e_{p, q}(x t) E_{p, q}(y t) \\
& =\left(\frac{1}{1-z\left(e_{p, q}(t)-1\right)}\right) e_{p, q}(x t) E_{p, q}(y t) \frac{[2]_{p, q} t}{e_{p, q}(t)+1} \frac{e_{p, q}(t)+1}{[2]_{p, q} t} e_{p, q}(x t) E_{p, q}(y t) \\
& =\frac{1}{[2]_{p, q} t}\left[\sum_{n=0}^{\infty}\left(\sum_{k=0}^{n}\left(\begin{array}{c}
n \\
k
\end{array}\right)_{p, q} G_{n-k}(x ; p, q) p\left(\begin{array}{l}
k \\
2
\end{array}\right)\right) \frac{t^{n}}{[n]_{p, q} !}+\sum_{n=0}^{\infty} G_{n}(x ; p, q) \frac{t^{n}}{[n]_{p, q} !}\right] \\
& \times \sum_{n=0}^{\infty} F_{n}(0, y ; z: p, q) \frac{t^{n}}{[n]_{p, q} !} \\
& =\frac{1}{[2]_{p, q}} \sum_{n=0}^{\infty}\left[\sum_{s=0}^{n}\left(\begin{array}{c}
n \\
s
\end{array}\right)_{p, q} \sum_{k=0}^{s}\left(\begin{array}{c}
s \\
k
\end{array}\right)_{p, q} G_{s-k}(x ; p, q) p\left(\begin{array}{c}
n \\
2
\end{array}\right)+\sum_{s=0}^{n}\left(\begin{array}{c}
n \\
s
\end{array}\right)_{p, q} G_{s}(x ; p, q)\right] \\
& \times F_{n+1-s}(0 . y ; z: p, q) \frac{t^{n}}{[n+1]_{p, q} !} .
\end{aligned}
$$

Comparing the coefficients of $\frac{t^{n}}{[n]_{p, q} !}$, then we have the asserted result (4.3).

Theorem 4.4. For $n \geq 0$, the following formula for $(p, q)$-type Fubini polynomials holds true:

$$
F_{n}(x, y ; z: p, q)=\sum_{l=0}^{n}\left(\begin{array}{l}
n \\
l
\end{array}\right)_{p, q}\left(x+y_{p, q}^{n-l}\right) \sum_{k=0}^{l} z^{k} k ! S_{2}(l, k: p, q) .
$$

Proof. From (2.1), we have

$$
\begin{gathered}
\sum_{n=0}^{\infty} F_{n}(x, y ; z: p, q) \frac{t^{n}}{[n]_{p, q} !}=\frac{1}{1-z\left(e_{p, q}(t)-1\right)} e_{p, q}(x t) E_{p, q}(y t) \\
=e_{p, q}(x t) E_{p, q}(y t) \sum_{k=0}^{\infty} z^{k}\left(e_{p, q}(t)-1\right)^{k} \\
=e_{p, q}(x t) E_{p, q}(y t) \sum_{k=0}^{\infty} z^{k} \sum_{l=k}^{\infty} k ! S_{2}(l, k: p, q) \frac{t^{l}}{[l]_{p, q} !} \\
=\sum_{n=0}^{\infty}(x+y)_{p, q}^{n} \frac{t^{n}}{[n]_{p, q} !} \sum_{l=0}^{\infty} z^{k} \sum_{k=0}^{l} k ! S_{2}(l, k: p, q) \frac{t^{l}}{[l]_{p, q} !}
\end{gathered}
$$

Replacing $n$ by $n-l$ in above equation, we get

$$
\begin{gathered}
\sum_{n=0}^{\infty} F_{n}(x, y ; z: p, q) \frac{t^{n}}{[n]_{p, q} !} \\
=\sum_{n=0}^{\infty}\left(\sum_{l=0}^{n}\left(\begin{array}{c}
n \\
l
\end{array}\right)_{p, q}(x+y)_{p, q}^{n-l} \sum_{k=0}^{l} z^{k} k ! S_{2}(l, k: p, q)\right) \frac{t^{n}}{[n]_{p, q} !} .
\end{gathered}
$$

Comparing the coefficients of $\frac{t^{n}}{[n]_{p, q} !}$ in both sides, we get (4.4). 
Theorem 4.5. For $n \geq 0$, the following formula for $(p, q)$-type Fubini polynomials holds true:

$$
F_{n}(x+r, y ; z: p, q)=\sum_{l=0}^{n}\left(\begin{array}{l}
n \\
l
\end{array}\right)_{p, q}\left(x+y_{p, q}^{n-l}\right) \sum_{k=0}^{l} z^{k} k ! S_{2}(l+r, k+r) .
$$

Proof. Replacing $x$ by $x+r$ in (2.1), we have

$$
\begin{gathered}
\sum_{n=0}^{\infty} F_{n}(x+r, y ; z: p, q) \frac{t^{n}}{[n]_{p, q} !}=\frac{1}{1-z\left(e_{p, q}(t)-1\right)} e_{p, q}((x+r) t) E_{p, q}(y t) \\
=e_{p, q}(x t) E_{p, q}(y t) e_{p, q}(r t) \sum_{k=0}^{\infty} z^{k}\left(e_{p, q}(t)-1\right)^{k} \\
=e_{p, q}(x t) E_{p, q}(y t) e_{p, q}(r t) \sum_{k=0}^{\infty} z^{k} \sum_{l=k}^{\infty} k ! S_{2}(l, k: p, q) \frac{t^{l}}{[l]_{p, q} !} \\
=\sum_{n=0}^{\infty}(x+y)_{p, q}^{n} \frac{t^{n}}{[n]_{p, q} !} \sum_{l=0}^{\infty} z^{k} \sum_{k=0}^{l} k ! S_{2}(l+r, k+r: p, q) \frac{t^{l}}{[l]_{p, q} !}
\end{gathered}
$$

Replacing $n$ by $n-l$ in above equation, we get

$$
\begin{gathered}
\sum_{n=0}^{\infty} F_{n}(x+r, y ; z: p, q) \frac{t^{n}}{[n]_{p, q} !} \\
=\sum_{n=0}^{\infty}\left(\sum_{l=0}^{n}\left(\begin{array}{c}
n \\
l
\end{array}\right)_{p, q}(x+y)_{p, q}^{n-l} \sum_{k=0}^{l} z^{k} k ! S_{2}(l+r, k+r: p, q)\right) \frac{t^{n}}{[n]_{p, q} !} .
\end{gathered}
$$

Comparing the coefficients of $\frac{t^{n}}{[n]_{p, q} !}$ in both sides, we get (4.5).

\section{References}

[1] Boyadzhiev, K. N., A series transformation formula and related polynomials, Int. J. Math. Math. Sci., 23(2005), 3849-3866.

[2] Carlitz, L., Eulerian numbers and polynomials, Mat. Mag., 32(1959), 164171.

[3] Corcino, R. B., On $p, q$-binomial coefficients, Electron, J. Combin. Number Theory, V.8, (2008), $\neq$ A29.

[4] Dil, A., Kurt, V, Investing geometric and exponential polynomials with EulerSeidel matrices, J. Integer Sequences, 14(2011), 1-12.

[5] Duran, U., Acikgoz, M., Araci, S., On higher order $(p, q)$-Frobenius-Euler polynomials, TWMS J. Pure. Appl. Math., 8(2)(2017), 198-208.

[6] Duran, U., Acikgoz, M., Apostal type $(p, q)$-Frobenious-Euler polynomials and numbers, Kragujevac J. Math., 42(4)(2018), 555-567.

[7] Duran, U., Acikgoz, M., Apostal type $(p, q)$-Bernoulli, $(p, q)$-Euler and $(p, q)$ Genocchi polynomials and numbers, Comput. Appl. Math., 8(1)(2017), 7-30.

[8] Duran, U., Acikgoz, M., Araci, S., On $(p, q)$-Bernoulli, $(p, q)$-Euler and $(p, q)$ Genocchi polynomials, J. Comput, Theor. Nanosci., 13(2016), 7833-7908.

[9] Gupta, V., $(p, q)$-Baskakov-Kontorovich operators, Appl. Math. INF. Sci., 10(4)(2016), 1551-1556.

[10] Gupta, V., Aral, A., Bernstein Durrmeyer operators and based on two parameters, Facta Universitatis (Nis), Ser. Math. Inform., 31(1)(2016), 79-95. 
[11] Graham, R. L., Knuth, D. E., Patashnik, O., Concrete Mathematics, AddisonWesley Publ. Co., New York, 1994.

[12] Kargin, L., Some formulae for products of Fubini polynomials with applications, arXiv:1701.01023v1[math.CA] 23 Dec 2016.

[13] Kurt, V., Simsek, Y., On the generalized Apostal type Frobenius Euler polynomials, Advances in difference equations, (2013)(1)(2013), '1-9.

[14] Kurt, B., A note on the Apostal type $q$-Frobenius Euler polynomials and generalizations of the Srivastava-Pinter addition theorems, Filomat, 30(1)(2016), 65-72.

[15] Milovanovic, G. V., Gupta, V., Malik, N., $(p, q)$-Beta functions and applications in approximation, Bol. Soc. Mat. Mex., 2016, doi: 10.1007/s0590-0160139-1.

[16] Pathan, M. A., Khan, W. A., Some implicit summation formulas and symmetric identities for the generalized Hermite-Bernoulli polynomials, Mediterr. J. Math., 12(2015), 679-695.

[17] Pathan, M. A., Khan, W. A., A new class of generalized polynomials associated with Hermite and Euler polynomials, Mediterr. J. Math., 13(3)(2016), 913-928.

[18] Simsek, Y., Generating functions for $q$-Apostal type Frobenius-Euler number and polynomials, Axioms, 1(2012), 395-403.

[19] Srivastava, H. M., and Manocha, H. L., A treatise on generating functions, Ellis Horwood Limited. Co. New York, 1984.

[20] Sadjang, P. N., On the fundamental theorem of $(p, q)$-calculus and some $(p, q)$ Taylor formulas, Results Math., (To appear).

[21] Tanny, S. M., On some numbers related to Bell numbers, Canad. Math. Bull., 17(1974), 733-738. 